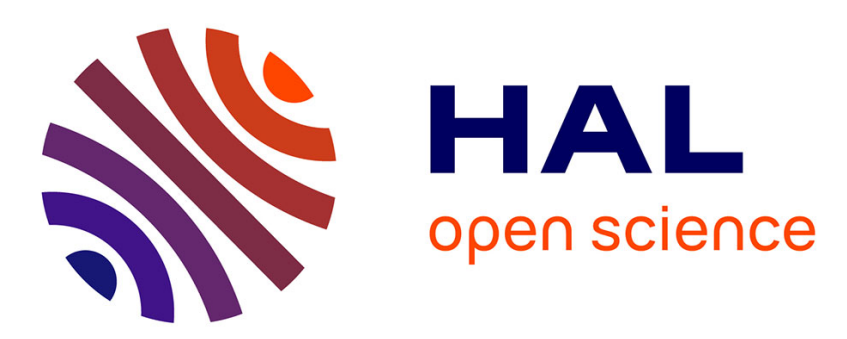

\title{
DIFFERENTIATED MULTIUSER RESOURCE ALLOCATION SCHEME FOR MULTI-BAND UWB SYSTEMS
}

Ayman Khalil, Matthieu Crussière, Jean-François Hélard

\section{- To cite this version:}

Ayman Khalil, Matthieu Crussière, Jean-François Hélard. DIFFERENTIATED MULTIUSER RESOURCE ALLOCATION SCHEME FOR MULTI-BAND UWB SYSTEMS. Signal Processing Advances in wireless Communications, Jun 2009, Italy. pp.701-711, 10.1109/SPAWC.2009.5161877. hal-00406391

\section{HAL Id: hal-00406391 \\ https://hal.science/hal-00406391}

Submitted on 22 Jul 2009

HAL is a multi-disciplinary open access archive for the deposit and dissemination of scientific research documents, whether they are published or not. The documents may come from teaching and research institutions in France or abroad, or from public or private research centers.
L'archive ouverte pluridisciplinaire HAL, est destinée au dépôt et à la diffusion de documents scientifiques de niveau recherche, publiés ou non, émanant des établissements d'enseignement et de recherche français ou étrangers, des laboratoires publics ou privés. 


\title{
DIFFERENTIATED MULTIUSER RESOURCE ALLOCATION SCHEME FOR MULTI- BAND UWB SYSTEMS
}

\author{
Ayman Khalil, Matthieu Crussière and Jean-François Hélard \\ Institute of Electronics and Telecommunications of Rennes (IETR) \\ INSA, 20 avenue des Buttes de Coësmes, 35043 Rennes, France \\ ayman.khalil@,insa-rennes.fr
}

\begin{abstract}
This paper considers the dynamic resource allocation in a multiuser context taking into account the quality of service (QoS) requirements for the high-rate ultra-wideband (UWB) systems. The aim of this paper is twofold. First, we exploit the effective SINR method in the multi-band WiMedia solution proposed for UWB systems. This method is based on the exploitation of the characteristics of multiple sub-carrier SINRs in order to provide the channel state information needed for the multiuser sub-band allocation. Second, using the effective SINR as a channel metric, we derive a multiuser convex optimization problem to find the optimal allocation for all users while differentiating between two traffic classes: hard-QoS and soft-QoS. A low-complexity cross-layer allocation algorithm is also proposed. The cross-layer solution is a combination of two simple processes, one working at the PHY level and the other at the MAC level. Simulation results show that the proposed cross-layer solution performance is close to that of the optimal solution and it outperforms the single-user WiMedia solution in the hard-QoS users case.
\end{abstract}

Index Terms - UWB, multi-band, effective SINR, QoS, cross-layer.

\section{INTRODUCTION}

Ultra-Wideband (UWB) is an emerging technology that offers great promises for the future low cost and high-rate wireless home networks. In 2002, the Federal Communications Commission (FCC) regulated UWB systems by allocating the 3.1 to $10.6 \mathrm{GHz}$ spectrum for unlicensed use of UWB [1]. In order to reduce interference with other existing systems, the FCC imposed a power spectral density (PSD) limit of -41.3 $\mathrm{dBm} / \mathrm{MHz}$.

The IEEE 802.15.3a wireless personal area networks (WPAN) standardization group defined a very high data rate physical layer based on UWB signalling. One of the multiple access techniques considered by the group is a multi-band orthogonal frequency division multiplexing (MB-OFDM) supported by the MultiBand OFDM Alliance (MBOA) and the WiMedia forum which merged in March 2005 and are today known as WiMedia Alliance [2].
WiMedia solution does not cope with multiuser access schemes and to this date, studies are focused on single-user access using the time-frequency codes (TFC) [2]. The aim of the paper is to study the WiMedia solution in a multiple access environment in order to achieve an efficient multiuser sub-band allocation based on a service differentiation approach and to satisfy existing users QoS requirements.

Most previous studies on resource allocation in multiuser OFDM systems provide optimal and suboptimal solutions without taking into account the QoS requirements and service differentiation issue. Two classes of optimization techniques have been proposed for the dynamic multiuser OFDM systems: margin adaptive (MA) and rate adaptive (RA). The MA objective is to minimize the overall transmit power given the constraints on the users data rate while the RA objective is to maximize the users rate with a total transmit power constraint. In [3], the authors use the MA and propose a multiuser OFDM subcarrier, bit, and power allocation algorithm to minimize the total transmit power. In [4], the authors use the RA and derive a multiuser convex optimization problem to find the optimal allocation of subchannels and then propose a low-complexity suboptimal solution. On the other hand, there have been some studies on resource allocation for OFDM-UWB systems but without considering the multiuser context [6], [7].

Our work consists in finding the optimal solution for the multiuser sub-band allocation for the WiMedia solution and to propose a low-complexity cross-layer solution based on the combination of the PHY and MAC layers functionalities. Hence, we propose first to exploit the effective SINR method proposed recently in the 3GPP standardization which can be effectively used in multi-band OFDM systems in order to compute an adequate metric for the channel information. We propose also to classify the applications into two service classes; the first is the hard-QoS class for applications such as voice transmission and real-time video streaming that have strict QoS constraints, and the second is the soft-QoS class for applications such as file transfer and email services that have tolerance for some requirements. Thus, the main objective is to find an optimal allocation that maximizes the sum-rate of the soft-QoS users while maintaining certain data rate level for the hard-QoS rates.

The remainder of this paper is organized as follows. Section 2 introduces the system model by presenting its 
characteristics. Section 3 derives the problem formulation as a convex optimization problem. In section 4 , we give the proposed low-complexity cross-layer solution. Section 5 presents simulation results showing the comparison between the proposed scheme and the optimal solution, and the performance of the multiuser solution compared to the singleuser WiMedia solution. Finally, section 6 concludes this paper.

\section{SYSTEM MODEL}

The WiMedia solution consists in combining OFDM with a multi-banding technique that divides the available band into 14 sub-bands of $528 \mathrm{MHz}$, as illustrated in Fig. 1. An OFDM signal can be transmitted on each sub-band using a 128-point inverse fast Fourrier transform (IFFT). Out of the 128 subcarriers used, only 100 are assigned to transmit data. Different data rates from 53.3 to $480 \mathrm{Mbps}$ are obtained through the use of forward error correction (FEC), frequencydomain spreading (FDS) and time-domain spreading (TDS), as presented in Table 1. The constellation applied to the different subcarriers is either a quadrature phase-shift keying (QPSK) for the low data rates or a dual carrier modulation (DCM) for the high data rates. Time-frequency codes (TFC) are used to provide frequency hopping from a sub-band to another at the end of each OFDM symbol. TFC allows every user to benefit from frequency diversity over a bandwidth equal to the three sub-bands of one channel.

The WiMedia solution offers potential advantages for highrate UWB applications, such as the signal robustness against channel selectivity and the efficient exploitation of the energy of every signal received within the prefix margin.

\section{PROBLEM FORMULATION}

\subsection{Channel Information}

In WiMedia solution as described in the previous section, each channel is divided into three sub-bands and the allocation is made by sub-band. For instance, three users in one channel have to share the available three sub-bands. Hence, to provide the users channel powers in each sub-band in order to achieve an efficient sub-band allocation that respects each user PHY conditions, the channel information is

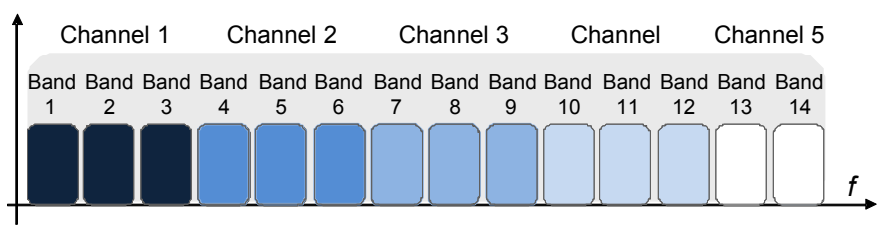

Figure 1. Channel distribution for WiMedia solution.
Table 1

WiMedia system data rates

\begin{tabular}{cccccc}
\hline $\begin{array}{c}\text { Data } \\
\text { Rate } \\
(\mathrm{Mbps})\end{array}$ & Modulation & $\begin{array}{c}\text { Coding } \\
\text { Rate }\end{array}$ & FDS & TDS & $\boldsymbol{\lambda}$ \\
\hline 53.3 & QPSK & $1 / 3$ & Yes & Yes & $\mathbf{1 . 4 9}$ \\
80 & QPSK & $1 / 2$ & Yes & Yes & $\mathbf{1 . 5 7}$ \\
110 & QPSK & $11 / 32$ & No & Yes & $\mathbf{1 . 5 2}$ \\
160 & QPSK & $1 / 2$ & No & Yes & $\mathbf{1 . 5 7}$ \\
200 & QPSK & $5 / 8$ & No & No & $\mathbf{1 . 8 2}$ \\
320 & DCM & $1 / 2$ & No & No & $\mathbf{1 . 8 5}$ \\
400 & DCM & $5 / 8$ & No & No & $\mathbf{1 . 8 2}$ \\
480 & DCM & $3 / 4$ & No & No & $\mathbf{1 . 8 0}$ \\
\hline
\end{tabular}

needed at the transmitter side. To do so, we propose to use the effective SINR method to represent the characteristics of each sub-band and to evaluate the system level performance after channel decoding in term of BER.

The effective SINR method consists in finding a compression function that maps the sequence of varying SINRs to a single value that is strongly correlated with the actual BER [8]. It is given by

$$
\operatorname{SINR}_{\text {eff }}=I^{-1}\left[\frac{1}{N} \sum_{i=1}^{N} I\left(\operatorname{SINR}_{i}\right)\right]
$$

In the effective SINR method, the following information measure function $I(x)$ is used

$$
I(x)=\exp \left(-\frac{x}{\lambda}\right)
$$

The inverse function of $I(x)$

$$
I^{-1}(x)=-\lambda \ln (x)
$$

Thus,

$$
\operatorname{SINR}_{e f f}=-\lambda \ln \left[\frac{1}{N} \sum_{i=1}^{N} \exp \left(-\frac{S I N R_{i}}{\lambda}\right)\right]
$$

where $N$ is the number of subcarriers in a sub-band, $\operatorname{SINR}_{i}$ is the ratio of signal to interference and noise for the $i^{\text {th }}$ subcarrier, and $\lambda$ is a scaling factor that depends on the selected modulation and coding scheme (MCS). $\lambda$ is computed and evaluated for the eight data rate modes as shown in Table 1.

\subsection{Problem Formulation and Solution}

The system consists of $K$ users where the first $K_{h}$ users are hard-QoS users and the remaining $K-K_{h}$ are soft-QoS users. The rate of a user $k$ in a sub-band $b$ is defined as

$$
r_{k, b}=\log _{2}\left(1+P_{k, b} \xi_{k, b}\right)
$$


where $P_{k, b}$ is the allocated power of user $k$ in the sub-band $b$, and $\xi_{k, b}$ is the effective SINR of user $k$ in this sub-band.

The objective in this paper is to optimize the sub-band allocation under the total power constraint $P_{T}$ so as to maximize the total data rate of $K-K_{h}$ soft-QoS users while maintaining a certain level of transmission rate for the $K_{h}$ hard-QoS users. The problem can be formulated as

$$
\begin{array}{cc}
\max _{S_{k}} & \sum_{k=K_{h}+1}^{K} \sum_{b \in S_{k}} r_{k, b} \\
\text { subject to } & \sum_{b \in S_{k}} r_{k, b} \geq R_{k}, \quad k=1, \ldots, K_{h} \\
& \sum_{k=1}^{K} \sum_{b=1}^{B} P_{k, b} \leq P_{T}
\end{array}
$$

where $B$ is total number of sub-bands, $R_{k}$ is the hard-QoS users required data rate, $S_{k}$ is the set of sub-bands assigned to user $k$. In our case, $S_{1}, S_{2}, \ldots, S_{k}$ are disjoint and each user is assigned one sub-band during one time interval. The formulated problem is a mixed integer programming problem which is hard to solve. However, we can convert this problem into a convex optimization problem by adopting a new parameter $\omega_{k, b}$ as proposed in [5]. It represents a time-sharing factor for the user $k$ of the sub-band $b$. The optimization problem can be reformulated as

$$
\begin{aligned}
\max _{\omega_{k, b}} & \sum_{k=K_{h}+1}^{K} \sum_{b=1}^{B} \omega_{k, b} \log _{2}\left(1+\frac{P_{k, b} \xi_{k, b}}{\omega_{k, b}}\right) \\
\text { subject to } & \sum_{b=1}^{B} \omega_{k, b} \log _{2}\left(1+\frac{P_{k, b} \xi_{k, b}}{\omega_{k, b}}\right) \geq R_{k}, \quad k=1, \ldots . . K_{h} \\
& \sum_{k=1}^{K} \omega_{k, b}=1 \quad \forall b \quad 0 \leq \omega_{k, b} \leq 1 \quad \forall k, b \\
& \sum_{k=1}^{K} \sum_{b=1}^{B} P_{k, b} \leq P_{T}
\end{aligned}
$$

The problem in (7) is a convex maximization problem. Using standard optimization techniques, we obtain the Lagrangian

$$
\begin{aligned}
L= & \sum_{k=K_{h}+1}^{K} \sum_{b=1}^{B} \omega_{k, b} \log _{2}\left(1+\frac{P_{k, b} \xi_{k, b}}{\omega_{k, b}}\right)+ \\
& \sum_{k=1}^{K_{h}} \alpha_{k}\left(\sum_{b=1}^{B} \omega_{k, b} \log _{2}\left(1+\frac{P_{k, b} \xi_{k, b}}{\omega_{k, b}}\right)-R_{k}\right)+ \\
& \sum_{b=1}^{B} \beta_{b}\left(1-\sum_{k=1}^{K} \omega_{k, b}\right)+\gamma\left(P_{T}-\sum_{k=1}^{K} \sum_{b=1}^{B} P_{k, b}\right)
\end{aligned}
$$

Let $P_{k, b}^{*}$ and $\omega_{k, b}^{*}$ be the optimal solution. After differentiating (8) with respect to $P_{k, b}$ by KKT optimality condition [9], we obtain

$$
\begin{array}{ll}
P_{k, b}^{*}=\omega_{k, b}\left(\frac{\alpha_{k}}{\gamma \ln 2}-\frac{1}{\xi_{k, b}}\right) & \text { for } k=1, . ., K_{h} \\
P_{k, b}^{*}=\omega_{k, b}\left(\frac{1}{\gamma \ln 2}-\frac{1}{\xi_{k, b}}\right) & \text { for } k=K_{h}+1, . ., K
\end{array}
$$

Then, after differentiating (8) with respect to $\omega_{k, b}$ we obtain

$$
\begin{aligned}
& \alpha_{k}\left[\log _{2}\left(\frac{\alpha_{k} \xi_{k, b}}{\gamma \ln 2}\right)-\frac{1}{\ln 2}\left(1-\frac{\gamma \ln 2}{\alpha_{k} \xi_{k, b}}\right)\right]-\beta_{b}=0 \\
& \text { for } k=1, . ., K_{h} \\
& \log _{2}\left(\frac{\xi_{k, b}}{\gamma \ln 2}\right)-\frac{1}{\ln 2}\left(1-\frac{\gamma \ln 2}{\xi_{k, b}}\right)-\beta_{b}=0 \\
& \text { for } k=K_{h}+1, . ., K
\end{aligned}
$$

Since $\omega_{k, b}^{*}$ should satisfy the following KKT condition

$$
\frac{\partial L}{\partial \omega_{k, b}^{*}}= \begin{cases}>0 & \omega_{k, b}^{*}=1 \\ =0 & \left.\omega_{k, b}^{*} \in\right] 0,1[ \\ <0 & \omega_{k, b}^{*}=0\end{cases}
$$

Substituting (11) and (12) into (13), we get

$$
\omega_{k, b}^{*}=\left\{\begin{array}{cc}
1 & I_{k, b}>\beta_{b} \\
0 & I_{k, b}<\beta_{b}
\end{array}\right.
$$

where $I_{k, b}$ is defined as

$$
\begin{aligned}
& I_{k, b}=\alpha_{k}\left[\log _{2}\left(\frac{\alpha_{k} \xi_{k, b}}{\gamma \ln 2}\right)-\frac{1}{\ln 2}\left(1-\frac{\gamma \ln 2}{\alpha_{k} \xi_{k, b}}\right)\right] \\
& \text { for } k=1, . ., K_{h} \\
& I_{k, b}=\log _{2}\left(\frac{\xi_{k, b}}{\gamma \ln 2}\right)-\frac{1}{\ln 2}\left(1-\frac{\gamma \ln 2}{\xi_{k, b}}\right) \\
& \text { for } k=K_{h}+1, . ., K
\end{aligned}
$$

We conclude that only the user with the largest $I_{k, b}$ can use the sub-band. In other words, for a sub-band $b$, if $I_{k, b}$ are different for all $k$, then

$$
\omega_{k^{\prime}, b}^{*}=1, \quad \omega_{k, b}^{*}=0 \quad \text { for all } k \neq k^{\prime}
$$

where

$$
k^{\prime}=\arg \max _{k} I_{k, b}
$$


In order to compute $I_{k, b}$ for all users, we need to find the set of $\alpha_{k}$ such that the hard-QoS rate constraints are satisfied.

Thus, an iterative searching algorithm is defined. We start with small values of $\alpha_{k}$ and we increase them with an iterative procedure until the data rate for all hard-QoS users are satisfied. However, this iterative solution requires an intensive computation cost. Therefore, we will propose in the next section a suboptimal solution based on a simple crosslayer approach.

\section{CROSS-LAYER SOLUTION}

The main parameters of the allocation function $I_{k, b}$ are the effective SINR $\xi_{k, b}$ and the Lagrange coefficient $\alpha_{k}$ which depends on the hard-QoS users only. Note that this function is monotonically increasing with respect to these parameters. As mentioned before, the determination of $\alpha_{k}$ requires a considerable complexity. Thus, we propose to define a new parameter which has the same properties of $\alpha_{k}$ but easier to compute. This new parameter is defined in the MAC layer which is responsible for QoS and service differentiation. Therefore, we define a traffic differentiation entity which is based on the weight or priority level concept. Consequently, each user $k$ is assigned a weight $q_{k}$ that depends on its requirements in terms of data rate and error rate and on its service class. For instance, hard-QoS users should be assigned a weight greater than the one assigned to the soft-QoS users.

Therefore, we define a cross-layer function as an allocation level $(A L)$ function that allocates the available subbands in a priority-based approach. This new allocation function combines the channel quality information through the effective SINR provided by the PHY layer with the service quality information through the weight or priority level provided by the defined MAC entity in a simple linear form. The allocation level function of user $k$ is given by

$$
A L_{k, b}=q_{k} \times \xi_{k, b}
$$

The weight $q_{k}$ is assigned in a way that respects the following condition

$$
\sum_{k=1}^{K} q_{k}=1
$$

For instance, a hard-QoS user may have a weight $q=0.6$ while two soft-QoS users may have each a weight $q=0.2$.

Consequently, the user $k^{\prime}$ is assigned the sub-band $b$ if he satisfies the condition

$$
k^{\prime}=\arg \max _{k} A L_{k, b}
$$

This new cross-layer allocation viewed as a suboptimal solution reduces significantly the complexity of the iterative procedure. Its efficiency is supported by simulation results in the next section.

\section{SYSTEM PERFORMANCE}

\subsection{Channel Model}

The channel used in this study is the one adopted by the IEEE 802.15.3a committee for the evaluation of UWB physical layer proposals [10]. This model is a modified version of Saleh-Valenzuela model for indoor channels [11], fitting the properties of UWB channels. Four different channel models (CM1 to CM4) are defined for the UWB system modelling, each with arrival rates and decay factors chosen to match different usage scenarios and to fit line-of-sight (LOS) and non-line-of-sight (NLOS) cases.

\subsection{Simulation Results}

In this section, we present the simulation results for the proposed cross-layer allocation solution and we compare it to the optimal solution as well as to the single-user WiMedia solution using TFC. Therefore, we use the proposed WiMedia data rates (see Table 1). The results are performed on the first three WiMedia sub-bands $(3.1-4.7 \mathrm{GHz})$ for CM1 channel model.

In Fig. 2, we present the case of three users transmitting simultaneously in the first WiMedia channel. The three users are assigned different data rates in order to show the advantage of hard-QoS users on soft-QoS users in term of error rate. We have one hard-QoS user transmitting at the highest rate, i.e. $480 \mathrm{Mbps}$ with a weight $q=0.6$, and two softQoS users transmitting at $400 \mathrm{Mbps}$ having each a weight $q=0.2$. We can see that the hard-QoS user outperforms the soft-QoS users with a considerable gain although it is transmitting at a higher rate. Hence, this gain proves that the proposed scheme can guarantee a higher performance for strict QoS users even if they have strict requirements.

In Fig.3, we compare the performance of a hard-QoS user transmitting at a rate of $320 \mathrm{Mbps}$ in the proposed cross-layer solution to that in the optimal solution and to the single-user WiMedia solution with TFC. Note that for the single-user solution TFC is exploited because it offers better performance. As shown in the figure, for a $B E R=10^{-4}$, the cross-layer and the optimal solutions are too close and offer a $2.5 \mathrm{~dB}$ gain for the hard-QoS user compared to WiMedia solution.

In Fig.4, we consider the case of soft-QoS users transmitting at a rate of $200 \mathrm{Mbps}$ and present their performance in the optimal and cross-layer solutions. For a $B E R=10^{-4}$, the optimal solution offers a $0.5 \mathrm{~dB}$ compared to the cross-layer solution. 


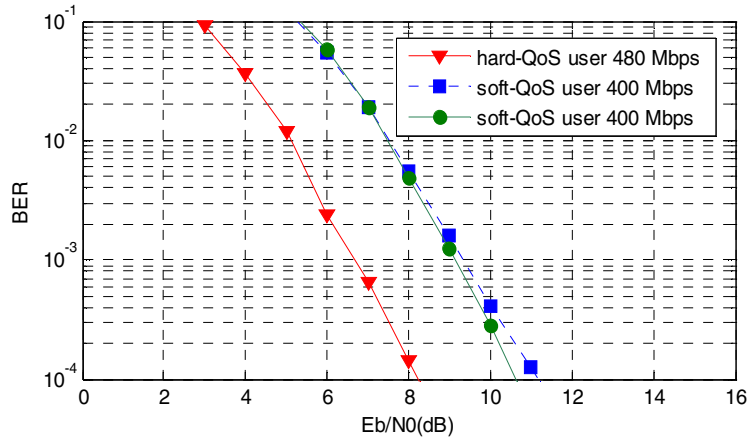

Figure 2. Performance of the cross-layer solution.

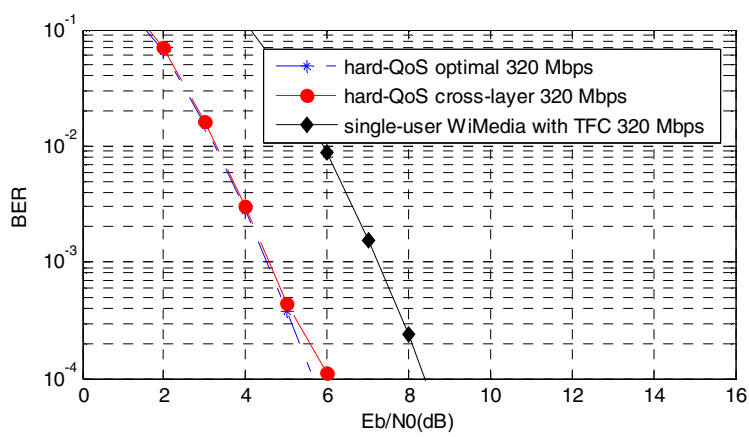

Figure 3. Hard-QoS users performance comparison.

On the other hand, we note that the performance of the crosslayer in the case of soft-QoS users is close to that of the single-user WiMedia solution. This proves that the performance of the multiuser cross-layer solution performance is never degraded compared to the single-user WiMedia solution.

\section{CONCLUSION}

In this paper, we considered the OFDM-UWB transmission in a multiuser context under QoS requirements. We exploited the effective SINR method to represent the channel power in a compressed way. Furthermore, based on a service differentiation principle, we formulated the problem of maximizing the rate of soft-QoS users while guaranteeing a certain level of data rate for the hard-QoS users. We presented the optimal solution and a low-complexity suboptimal solution based on a cross-layer approach that exploits jointly QoS from MAC layer and channel information from PHY layer. We showed through simulations that the proposed cross-layer solution improves the performance of the hardQoS users in term of error rate without decreasing the performance of soft-QoS users. Moreover, we showed that the multiuser cross-layer solution and the multiuser optimal solution have a close performance while outperforming both the single-user WiMedia solution.

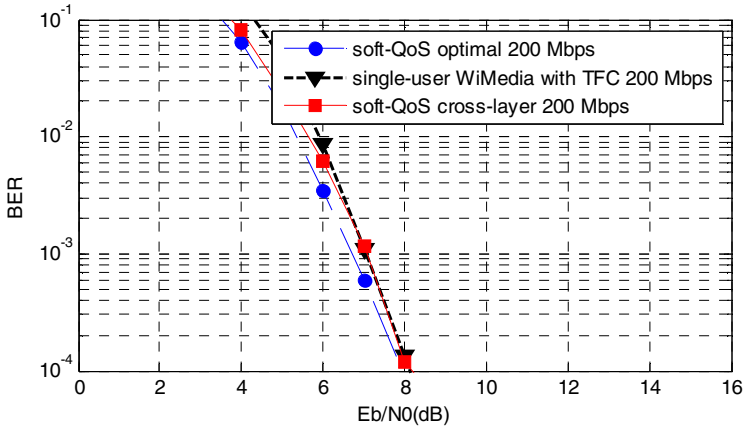

Figure 4. Soft-QoS users performance comparison.

\section{ACKNOWLEDGMENT}

The research leading to these results has received funding from the European Community's Seventh Framework Programme FP7/2007-2013 under grant agreement $n^{\circ} 213311$ also referred as OMEGA.

\section{REFERENCES}

[1] "First report and order, revision of part 15 of the commission's rules regarding ultra-wideband transmission systems," FCC, ET Docket 98-153, Feb. 14, 2002.

[2] Batra et al., "Multi-Band OFDM physical layer proposal for IEEE 802.15 task group 3a," IEEE document P802.1504/0493r1, Texas Instruments et al., Sept. 2004.

[3] C. Y. Wong, R. S. Cheng, K. B. Letaief, and R. D. Murch, "Multiuser OFDM with adaptive subcarrier, bit and power allocation," IEEE Journal on Selected Areas in Comm., vol. 21, no. 2, pp. 171-178, Feb.2003.

[4] W. Rhee and J. M. Cioffi, "Increase in capacity of multiuser OFDM system using dynamic subchannel allocation," in Proc. of IEEE VTC, 2000.

[5] Z. Shen, J. G. Andrews, and B. L. Evans, "Adaptive resource allocation in multiuser OFDM systems with proportional fairness," IEEE Trans. on Wireless Comm., vol. 4, no. 6, pp. 2726-2737, Nov. 2005.

[6] Z. Chen, D. Wang and G. Ding, "An OFDM-UWB Scheme with Adaptive Carrier Selection and Power Allocation," in Proc. IEEE Intern. Conference on Wireless Comm., Networking and Mobile Computing (WiCOM '06), pp.1-4, China, Sept. 2006.

[7] Z. Xu and L. Liu, "Power allocation for multi-band OFDM UWB communication networks," in Vehicular Technology Conference (VTC2004-Fall), vol. 1, pp. 368-372, Los Angeles, Sept. 2004.

[8] 3GPP TSG-RAN-1, "R1-030999: considerations on the systemperformance evaluation of HSDP using OFDM modulations"; RAN WG1 \#34.

[9] D.P Bertsekas, Nonlinear Programming $2^{\text {nd }}$ Edition. Athena Scientific, 1999.

[10] J. Foester, "Channel Modeling sub-committee report (final)," IEEE P802.15.-02/490rl-SG3a,2003.

[11] A. Saleh and R. Valenzuela, "A statistical model for indoor multipath propagation, "IEEE Journal on Selected Areas in Comm., vol. 5, no. 2, pp. 128-137, Feb. 1987. 